\title{
RASTREIO DE ESCOLIOSE NO ADOLESCENTE
}

Exma. Senhora Directora da Revista Portuguesa de Clínica Geral:

No artigo "Boletim de Saúde Infantil e Juvenil - o exame global de saúde dos 11 aos 13 anos (parte II)», ${ }^{1} e ́$ referida a importância da realização do rastreio de escoliose no exame global de saúde dos 11 aos 13 anos, através da realização, entre outros, da manobra de Adams. Gostaria, a este propósito, de fazer notar uma recomendação da United States Preventive Services Task Force, ${ }^{2}$ em que é referido que o rastreio da escoliose idiopática no adolescente não deve ser realizado (grau D) pois existe evidência de que os maleficios ultrapassam os potenciais beneficios.

Com os cumprimentos,

Nuno Machado*

*Médico interno em formação específica de Medicina Geral e Familiar.

Unidade de Saúde Familiar Aquae Flaviae, Chaves

\section{REFERÊNCIAS BIBLIOGRÁFICAS}

1. Freitas $C$, Sousa HS, Fonseca H. Boletim de Saúde Infantil e Juvenil - o exame global de saúde dos 11 aos 13 anos (parte II). Rev Port Clin Geral 2011 Mar-Abr; 27 (2): 193-202.

2. U.S. Preventive Services Task Force. Screening for idiopathic scoliosis in adolescents: recommendation statement. June 2004. Agency for Healthcare Research and Quality, Rockville, MD. Disponível em: http://www.uspreventiveservicestaskforce.org/3rduspstf/scoliosis/scoliors.htm [acedido em 30/05/2011].

\section{Resposta dos Autores}

Exma. Senhora Directora da Revista Portuguesa de Clínica Geral:

Agradeço o comentário feito pelo Dr. Nuno Machado relativamente ao nosso artigo «Boletim de Saúde Infantil e Juvenil - o exame global de saúde dos 11 aos 13 anos (parte II)», ${ }^{1}$ referindo a existência de evidência científica contra a realização do rastreio da escoliose idiopática no adolescente. Realçamos, no entanto, que a postura consta da listagem dos parâmetros a examinar na consulta dos 11-13 anos do Boletim de Saúde Infantil e Juvenil em vigor. Além disso, na referida recomendação está claramente expresso que «Although routine screening of adolescents for idiopathic scoliosis is not recommended, clinicians should be prepared to evaluate idiopathic scoliosis when it is discovered incidentally or when the adolescent or parent expresses concern about scoliosis." ${ }^{2}$

Pelos autores,

Helena Fonseca*

*Chefe de Serviço, Unidade de Medicina do Adolescente, Departamento da Criança e da Família, Hospital de Santa Maria

\section{REFERÊNCIAS BIBLIOGRÁFICAS}

1. Freitas $C$, Sousa HS, Fonseca H. Boletim de Saúde Infantil e Juvenil - o exame global de saúde dos 11 aos 13 anos (parte II). Rev Port Clin Geral 2011 Mar-Abr; 27 (2): 193-202.

2. U.S. Preventive Services Task Force. Screening for idiopathic scoliosis in adolescents: recommendation statement. June 2004. Agency for Healthcare Research and Quality, Rockville, MD. Disponível em: http://www.uspreventiveservicestaskforce.org/3rduspstf/scoliosis/scoliors.htm [acedido em 06/07/2011]. 